\title{
ON THE TRANSPOSE OF SIMPLE SETS OF POLYNOMIALS EFFECTIVE IN FABER REGIONS
}

\begin{abstract}
M. NASSIF
ABstract. The effectiveness properties, in a Faber region, of the transpose of simple absolutely monic sets of polynomials effective in the same region, are investigated in the present paper. A lower bound is calculated for an inevitable normalizing factor which ensures the finiteness of the Cannon function of the transpose of the normalized set in the region considered. Yet, except in the case of a circle with centre origin, no value of the normalizing factor can ensure the effectiveness of the transpose in the region considered.
\end{abstract}

1. Preliminaries. The transpose of a $U$-basic set of polynomials is the set whose matrix of coefficients is the transpose of that of the given set. ${ }^{1}$ When the given set is simple, absolutely monic and effective in the unit circle it can be shown that the transpose is effective in the same circle. It is proposed to investigate, in the present paper, the extent of generalization of this result when the unit circle is replaced by the Faber curve $C$. This is a simple closed regular curve which is the map in the $z$-plane of the circle $|t|=\gamma$, say, by the transformation ${ }^{2}$

$$
z=\phi(t)=t+\sum_{n=0}^{\infty} a_{n} t^{-n}
$$

which is supposed to be conformal in $|t|>T$ for some $T<\gamma$. Such

Received by the editors January 27, 1969.

AMS Subject Classifications. Primary 3030.

Key Words and Phrases. Expansion in polynomials, simple set of polynomials, Faber regions.

1 The reader is supposed to be acquainted with the theory of basic sets of polynomials as given by Whittaker [5], as well as the essentials of the theory of Faber regions as given by Faber [1], by Hassab-el-Nabi (cf. Whittaker [5, pp. 77-78]) and by Newns [2, pp. 452-453], [3, p. 190], [4, pp. 747-751]. The definition of $U$-basic sets as well as the above definition of the transpose are given by Newns [2, p. 443 and p. 455 respectively]. It is easy to see that a simple set is $U$-basic, as well as its transpose when it exists.

2 This "normalized" form of the transformation $z=\phi(t)$ adopted by Newns [4, p. 747] is found to be convenient to be applied in the present work; cf. formula (1.3) below. 
transformation maps the domain ${ }^{3} E(\gamma)$ onto $E(C)$ so that, for $T<r<\infty$, the circles $|t|=r$ are mapped on to the simple closed regular curves $\left(C_{r}\right)$.

Being simple in $|t|>T$, the function $z=\phi(t)$ has got an inverse $t=\psi(z)$, which is regular and one-to-one in the map of $E(T)$ in the $z$-plane.

The base for the class $H\left(C_{r}\right) ; r>T$, is the set $\left\{f_{n}(z)\right\}$ of Faber polynomials associated with the transformation $z=\phi(t)$, and is thus termed $\phi$-base (cf. Newns [4, p. 748]). These polynomials were defined by Faber $[1$, p. 391] through the generating function

$$
\frac{t \phi^{\prime}(t)}{\phi(t)-z}=\sum_{n=0}^{\infty} f_{n}(z) t^{-n}
$$

where

$$
f_{n}(z)=\sum_{k=0}^{n} f_{n, k} z^{k}, \quad f_{n, n}=1 \quad(n \geqq 0),
$$

and the following formula was established by Newns [4, p. 749].

$$
\begin{aligned}
f_{n}(z)=\{\psi(z)\}^{n}+(1 / 2 i \pi) & \int_{C_{T^{\prime}}} \frac{\{\psi(\xi)\}^{n} d \xi}{\xi-z} \\
& \left(z \in C_{r} ; r>T^{\prime}>T ; n \geqq 1\right) .
\end{aligned}
$$

It has been also shown by Newns $[4,(2.18)]$ that the inverse set $\left\{\bar{f}_{n}(z)\right\}$ is, in fact, the $\psi$-base. Thus, with analogy of the formula (1.4), the polynomials $\left\{\bar{f}_{n}(z)\right\}$ will accord to the relation

$$
\begin{aligned}
\bar{f}_{n}(z)=\{\phi(z)\}^{n}+(1 / 2 i \pi) \int_{|t|=T^{\prime}} & \frac{\{\phi(t)\}^{n} d t}{t-z} \\
& \left(T<T^{\prime}<|z| ; n \geqq 1\right) .
\end{aligned}
$$

2. The functions $\phi(t), \psi(z)$. In this section further study is carried out on the functions $\phi(t)$ and $\psi(z)$. To this end, we shall write

$$
\begin{array}{r}
G(r)=\sup \{|\phi(t)|:|t|=r\}, \quad g(r)=\inf \{|\phi(t)|:|t|=r\} \\
(r>T),
\end{array}
$$

3 Adopting Newns notation [2, p. 436], $D(C)$ and $E(C)$ denote respectively the domains interior and exterior to the closed curve $C$. The closure of $D(C)$ is denoted by $\bar{D}(C)$ and the class of functions regular in $D(C)$ is written $H(C)$. When $C$ is the circle $|z|=r$, the above entites are respectively referred to as $D(r), E(r), \bar{D}(r)$ and $H(r)$. 


$$
\begin{aligned}
T_{0} & =\inf \{|\phi(t)|:|t|>T\}=\inf \{g(r): r>T\}, \\
h(r) & =\sup \{|\psi(z)|:|z|=r\}=\sup \{|t|:|\phi(t)|=r\}
\end{aligned}
$$

and

$$
\rho_{0}=\inf \left\{h(r): r>T_{0}\right\} .
$$

The supremum in (2.3) is taken over those points of $|z|=r$ which lie in the domain of existence of $\psi(z)$. First of all, the nature of the mapping function necessitates that

(2.5) $G(r)$ is a strictly increasing function of $r$ in $T<r<\infty$.

Also, it can be deduced from Newns results [4, (2.12) and (2.14)] that $h(r)$ is monotonic increasing, hence the nonconstancy of $\psi(z)$ implies that

(2.6) $\quad h(r)$ is a strictly increasing function of $r$ in $T_{0}<r<\infty$.

Moreover, in view of (2.2) and (2.3), the numbers $\alpha$ and $\beta$ can be defined as follows:

$$
\alpha=G(\gamma)>T_{0}, \quad \beta=\alpha h(\alpha) .
$$

Whence, it follows from (2.2), (2.4) and (2.6) that

$$
T<\rho_{0}=h\left(T_{0}\right)<h(\alpha)=\beta / \alpha .
$$

Now, it can be deduced from Newns $[4,(2.10)]$ that $\phi(t) \neq 0$ for $|t|>\rho_{0}$. Hence, the function $\Omega(u)=1 / \phi(1 / u)$ is regular for $|u|<1 / \rho_{0}$. Consequently, $\sup \{|\Omega(u)|:|u|=1 / r\}=1 / g(r)$ is strictly decreasing for $1 / r<1 / \rho_{0}$. We have thus established the monotony property of $g(r)$ in the form

$$
g(r) \text { is a strictly increasing function of } r \text { in } \rho_{0}<r<\infty .
$$

The monotony properties (2.5) of $G(r),(2.6)$ of $h(r)$ and (2.9) of $g(r)$ will be assumed in the subsequent work without being explicitly stated.

The following lemma establishes the relation between the functions $h(r)$ and $g(r)$.

Lemma 1. If $\rho>\rho_{0}$ and $g(\rho)=r$ then $r>T_{0}$ and $h(r)=\rho$. Conversely, if $r>T_{0}$ and $h(r)=\rho$ then $\rho>\rho_{0}$ and $g(\rho)=r$.

Proof. For the first assertion, we observe from (2.2) and the first inequality in (2.8) that, if $\rho>\rho_{0}$, 


$$
r=g(\rho)>g\left(\rho_{0}\right)=\inf \left\{g(R): R>\rho_{0}>T\right\} \geqq T_{0} .
$$

Also, it is easily seen from the definition (2.1) of $g(r)$ and (2.3), that $h(r) \geqq \rho$. Moreover, if $t$ is any number for which $|t|=\rho^{\prime}>\rho$ then $|\phi(t)|>r$, so that $h(r) \leqq \rho$. Consequently, $h(r)=\rho$.

To prove the second assertion, we note that the inequality $\rho>\rho_{0}$ follows directly from the first equality in (2.8). Suppose now that $g(\rho)=r_{1}$, then by the first assertion we have $r_{1}>T_{0}$ and $h\left(r_{1}\right)=\rho$. Hence, $r_{1}=r$ and the lemma is proved.

3. Normalizing factor. Our concern in the present work is to discuss the effectiveness properties in $D(C)$ of the transpose of simple, absolutely monic sets ${ }^{4}\left\{p_{n}(z)\right\}$ of polynomials which are effective in $D(C)$. It should be first observed that the transpose of such sets may not exist in $D(C)$. This phenomenon is exhibited even by some $\phi-$ bases. In fact, the transpose ${ }^{5}\left\{\tilde{f}_{n}(z)\right\}$ of the $\phi$-base $\left\{f_{n}(z)\right\}$, which is simple monic set effective in $D(C)$, is obtained by differentiation of (1.2), in the form

$$
\tilde{f}_{n}(z)=\phi^{\prime}(1 / z) / z\{\phi(1 / z)\}^{n+1} \quad(n \geqq 0) .
$$

Thus the circle of regularity of $\bar{f}_{n}(z)$ is at most $|z|=1 / T$, and this circle does not cover $D(C)$ for certain ${ }^{6}$ forms of $C$. Therefore, to ensure the existence in $D(C)$ of the transpose of the set $\left\{p_{n}(z)\right\}$, the polynomials $\left\{p_{n}(z)\right\}$ have to be multiplied by certain coefficients before being exposed to our discussion. In fact, if $\left\{p_{n}(z)\right\}$ is the given set, the normalized set $\left\{P_{n}(z)\right\}$, whose transpose is to be considered in the present paper, is given by

$$
P_{n}(z)=\xi^{-n} p_{n}(z) \quad(n \geqq 0),
$$

where the finite number $\xi$ may assume any value $\geqq \beta$. The number ${ }^{i} \xi$ thus determined will be here referred to as the normalizing factor. This choice of normalizing factor yields favourable results concerning the effectiveness properties of the transpose $\left\{\widetilde{P}_{n}(z)\right\}$ in $D(C)$.

4 Throughout the work $\left\{p_{n}(z)\right\}$ will denote such sets.

${ }^{5}$ In our notation a tilde $(\sim)$ over a set or an entity indicates that either the set is or the entity belongs to the transpose of the corresponding set. A similarly situated bar (-) indicates the same for inverse sets.

${ }^{6}$ An obvious form is the Cassini oval $\left|z^{2}-1\right|=c^{2} ; c>1$.

${ }^{7}$ In what follows $\phi(t), T, \gamma, f_{n}(z), f_{n, k}, G(r), g(r), T_{0}, h(r), \rho_{0}, \alpha, \beta$ and $\xi$ will always denote the expressions or numbers given respectively in (1.1), (1.2), (1.3), (2.1), (2.2), (2.3), (2.4), (2.7) and (3.2). Also, $\psi(z)$ will always denote the inverse of the function $\phi(t)$. 
For if, on the one hand, the Faber curve $C$ is the circle $|z|=R$; $R>0$, then the set $\left\{P_{n}(z)\right\}$ of (3.2), will be defined by $P_{n}(z)=\xi^{-n} p_{n}(z)$; $\xi \geqq R^{2}$, whose transpose is easily shown to be effective in $D(R)$, whenever the set $\left\{p_{n}(z)\right\}$ is effective there.

On the other hand, if $C$ is not a circle with centre origin, as we shall always assume in the subsequent work, the normalizing factor $\xi$ will ensure the finiteness of the Cannon function ${ }^{8}$ of the transpose $\left\{\widetilde{P}_{n}(z)\right\}$ for the curve $C$. The precise meaning of this statement is illustrated in the following theorem.

THEOREM 1. Let $\left\{p_{n}(z)\right\}$ be a simple, absolutely monic set of polynomials which is effective in $D(C)$, and suppose that $\tilde{\kappa}(C)$ is the Cannon function for the curve $C$, of the transpose $\left\{\widetilde{P}_{n}(z)\right\}$ of the set $\left\{P_{n}(z)\right\}$, given by (3.2). Then $\tilde{\kappa}(C) \leqq \sigma$, where

$$
\sigma=h(\xi / \eta), \quad G(\eta)=g(\xi / \alpha) .
$$

Furthermore, the choice (2.7) of the lower bound $\beta$ for the normalizing factor $\xi$, is justified in the sense that any value of $\xi$ which is less than $\beta$ does not ensure that the transpose of the normalized set is even basic in $D(C)$. This fact is illustrated by the example immediately given below.

It is now natural to ask whether sufficiently large values of $\xi$ ensure the effectiveness in $D(C)$ of the transpose $\left\{\widetilde{P}_{n}(z)\right\}$. The answer is by no means positive according to Theorem 2 below, which asserts that, for all forms of the Faber curve $C$ there is a set $\left\{p_{n}(z)\right\}$ such that the normalized transpose $\left\{\widetilde{P}_{n}(z)\right\}$ is not effective in $D(C)$ for any $\xi \geqq \beta$.

4. Example. Let $C$ be the Cassini oval $\left|z^{2}-1\right|=c^{2} ; c>1$, for which the mapping function is

$$
\phi(t)=t\left(1+1 / t^{2}\right)^{1 / 2} \quad(|t|>1) .
$$

Applying (2.7) we obtain, by actual calculation from (4.1),

$$
\beta^{2}=\left(c^{2}+1\right)\left(c^{2}+2\right) .
$$

We shall therefore take $\xi$ such that

$$
0<\xi^{2}<\left(c^{2}+1\right)\left(c^{2}+2\right),
$$

and consider the following set $\left\{p_{n}(z)\right\}$,

$$
\begin{aligned}
p_{0}(z) & =f_{0}(z), \\
p_{2 n-1}(z) & =i^{2 n-1} f_{2 n-1}(z), \quad p_{2 n}(z)=(-1)^{n}\left\{\rho^{2 n}+f_{2 n}(z)\right\} \quad(n \geqq 1),
\end{aligned}
$$

${ }^{8}$ For definition of Cannon function of a set for a Faber curve, cf. Newns [2, p. 453]. 
where $\left\{f_{n}(z)\right\}$ is the $\phi$-base and the number $\rho$ is fixed as follows.

$$
0<\rho<c, \quad \xi^{2} /\left(c^{2}+1\right)<\rho^{2}+2 .
$$

The possibility of these inequalities follows from (4.2). It is easily seen that this set $\left\{p_{n}(z)\right\}$ is effective in $D(C)$, and in view of (3.1), (4.1) and (4.3), the transpose $\left\{\widetilde{P}_{n}(z)\right\}$ will be

$$
\begin{aligned}
& \widetilde{P}_{0}(z)=\xi^{2} /\left(\xi^{2}-z^{2}\right)-\rho^{2} z^{2} /\left(\xi^{2}+\rho^{2} z^{2}\right), \\
& \widetilde{P}_{n}(z)=\xi^{2}(i z)^{n}\left(\xi^{2}-z^{2}\right)^{-(n / 2)-1} \quad(n \geqq 1),
\end{aligned}
$$

provided that $|z|<\min (\xi, \xi / \rho)$. Moreover, when $|z|<\xi\left(2+\rho^{2}\right)^{-1 / 2}$, the following representation can be verified.

$$
\begin{aligned}
f_{2 n}(z)= & (-1)^{n}\left[\tilde{P}_{0}(z)+\sum_{k=1}^{\infty}\left\{2-\left(1+\rho^{2}\right)^{k}\right.\right. \\
& \left.\left.+\sum_{j=1}^{k^{\prime}}\left(\begin{array}{c}
n \\
j
\end{array}\right)\left(\begin{array}{l}
k \\
j
\end{array}\right) \xi^{2 j}\right\} \widetilde{P}_{2 k}(z)\right] \\
= & \sum_{k=0}^{\infty} \pi_{2 n, 2 k} \tilde{P}_{2 k}(z),
\end{aligned}
$$

say, where $k^{\prime}=\min .(k, n)$. Now, in view of (4.4) we can choose $\zeta \in D(C)$ real and satisfying the following inequalities.

$$
0<\zeta<\left(c^{2}+1\right)^{1 / 2}, \quad \xi^{2} /\left(2+\rho^{2}\right)<\zeta^{2}<\xi^{2} / 2 .
$$

Simple calculation based on (4.5), (4.6) and (4.7) leads to

$$
\begin{aligned}
\left|\pi_{2 n, 2 k} \widetilde{P}_{2 k}(\zeta)\right|> & \frac{\xi^{2}}{\xi^{2}-\zeta^{2}}\left(\frac{1+\rho^{2}}{\xi^{2} / \zeta^{2}-1}\right)^{k}-\frac{2 \xi^{2}}{\xi^{2}-\zeta^{2}} \\
& -\frac{\xi^{2}}{\xi^{2}-2 \zeta^{2}}\left(1+\frac{\xi^{2} \zeta^{2}}{\xi^{2}-2 \zeta^{2}}\right)^{n} \quad(k \geqq 1) .
\end{aligned}
$$

Since $\left(1+\rho^{2}\right) /\left(\xi^{2} / \zeta^{2}-1\right)>1$, then by taking $k$ large enough we can make

$$
\left|\pi_{2 n, 2 k} \widetilde{P}_{2 k}(\zeta)\right|>\frac{\xi^{2}}{2\left(\xi^{2}-\zeta^{2}\right)}\left(\frac{1+\rho^{2}}{\xi^{2} / \zeta^{2}-1}\right)^{k} .
$$

Hence, the representation (4.6) of $f_{2 n}(\zeta)$ is impossible and the set $\left\{\widetilde{P}_{n}(z)\right\}$ fails to be basic in $D(C)$.

5. Proof of Theorem 1. We first observe from (2.7) and Lemma 1, that $g(\beta / \alpha)=\alpha$. Since $\xi \geqq \beta$ it follows from (3.3) that

$$
G(\eta)=g(\xi / \alpha) \geqq g(\beta / \alpha)=\alpha=G(\gamma) .
$$


Hence the monotony and continuity of the function $G(r)$ ensure the existence of the number $\eta$ to satisfy

$$
T<\gamma \leqq \eta<\xi / \alpha .
$$

Whence

$$
\xi / \eta>\alpha>T_{0},
$$

and according to (2.3) the number $\sigma=h(\xi / \eta)$ is well defined.

Let $\left\{q_{n}(z)\right\}$ be the set associated with ${ }^{9}\left\{p_{n}(z)\right\}$ with respect to $\phi$. Then $\left\{q_{n}(z)\right\}$ is a simple, absolutely monic set of polynomials which is effective in $D(\gamma)$ and consequently in $D(\eta)$ and in $D(\xi / \alpha)$. Hence, the inverse set $\left\{\bar{q}_{n}(z)\right\}$, which is simple and absolutely monic, will be effective in $D(\eta)$.

Let $r$ be any number satisfying $T<r<\eta$ and write

$$
M_{n}(r)=\sup \left\{\left|q_{n}(z)\right|:|z|=r\right\}, \quad \mu(r)=\limsup _{n \rightarrow \infty}\left\{M_{n}(r)\right\}^{1 / n} .
$$

It follows therefore that a number $r^{\prime}$ can be chosen so that (cf. footnote 5)

$$
T<r<r^{\prime}<\eta, \quad \bar{\mu}(r) \leqq r^{\prime} .
$$

To proceed further we put $G\left(r^{\prime}\right)=R$ and $h(R)=\rho$, thus by (2.2), (2.3) and (5.3) and appealing to Lemma 1, it can be inferred that

$$
T<\rho_{0}<\rho<\xi / \alpha .
$$

Writing $\lambda\left\{x ;\left(y_{n}\right)\right\}$ for the Cannon function of the Cannon set $\left\{y_{n}(z)\right\}$ in $|z|=x$, then a combination of (1.4), (1.5), (5.3), and (5.4) can easily lead to the relation

$$
\lambda\left\{\bar{\mu}(r) ;\left(\bar{f}_{n}\right)\right\} \leqq \rho<\xi / \alpha .
$$

Now, it is seen that

$$
\left\{\bar{p}_{n}(z)\right\}=\left\{\bar{f}_{n}(z)\right\}\left\{\bar{q}_{n}(z)\right\} .
$$

Applying then Newns result [3, Theorem 3] concerning the product set (5.6) and the relation (5.5) and recalling that $\left\{q_{n}(z)\right\}$ is effective in $D(\xi / \alpha)$ we can easily arrive at the relation

$$
\lambda\left\{r+;\left(p_{n}\right)\right\} \leqq \mu(\rho)<\xi / \alpha .
$$

That is to say, the set $\left\{p_{n}(z)\right\}$ is effective for $H(\xi / \alpha)$ in $^{10} D_{+}(r)$. It

${ }^{9}$ For definition and properties of associated sets cf. Newns [3, p. 190].

${ }^{10}$ In our notation $D_{+}$denotes some unspecified domain containing $\bar{D}$, and $\bar{H}$ stands for the class of functions regular in some $D_{+}$, cf. Newns [2, p. 436]. 
follows that the transposed inverse set, which is the set $\left\{\tilde{p}_{n}(z)\right\}$, will be effective for $H(1 / r)$ in $D_{+}(\alpha / \xi)$. Observing that our transpose $\left\{\widetilde{P}_{n}(z)\right\}$ is, in fact, the set $\left\{\tilde{p}_{n}(z / \xi)\right\}$, we deduce that the transpose is effective for $H(\xi / r)$ in $D_{+}(\alpha)$. Since $r$ is arbitrary chosen near to $\eta$, we conclude that the transpose $\left\{\widetilde{P}_{n}(z)\right\}$ is effective tor $\bar{H}(\xi / \eta)$ in $D_{+}(\alpha)$. Finally, observing that $\bar{D}(\xi / \eta) \subset \bar{D}\left(C_{\sigma}\right)$ (cf. Newns [4, (2.10)]) and that $\bar{D}(C) \subset D_{+}(\alpha)$, it follows that $\left\{\widetilde{P}_{n}(z)\right\}$ is effective for $\bar{H}\left(C_{\sigma}\right)$ in $\bar{D}(C)$; or equivalently $\tilde{\kappa}(C) \leqq \sigma$. Theorem 1 is therefore established.

It should be observed that, if $\xi>\beta$, and if the set $\left\{p_{n}(z)\right\}$ is assumed to be only effective in $\bar{D}(C)$, then the associated set $\left\{q_{n}(z)\right\}$ will be effective in $D(\eta)$ and in $D(\xi / \alpha)$ and the above procedure leads to the same result. Hence, we have the following corollary.

Corollary. Let $\left\{p_{n}(z)\right\}$ be a simple absolutely monic set of polynomials which is effective in $\bar{D}(C)$, and let $\tilde{\kappa}(C)$ be as in Theorem 1 , where $\xi>\beta$. Then we still have $\tilde{\kappa}(C) \leqq \sigma$.

6. Noneffectiveness of the transpose. We propose here to fulfill the statement given at the end of $\$ 3$. To this aim we first observe from (2.7) that there is a point $z_{0}$ on the Faber curve $C$ such that

$$
\left|z_{0}\right|=\alpha \text {. }
$$

Therefore, by the definition (3.3), two real numbers $\delta$ and $\omega$ can be so chosen that

$$
\left|\phi\left(\xi e^{-i \delta} / z_{0}\right)\right|=g(\xi / \alpha)=G(\eta)=\left|\phi\left(\eta e^{-i \omega}\right)\right|=\tau,
$$

say. Consider now the set $\left\{p_{n}(z)\right\}$ given by

$$
p_{n}(z)=e^{i n \delta} f_{n}(z) \quad(n \geqq 0) .
$$

Being effectively equivalent to the $\phi$-base $\left\{f_{n}(z)\right\}$, the set $\left\{p_{n}(z)\right\}$ is simple, absolutely monic and effective in $D(C)$. According to (3.2), the normalized set $\left\{P_{n}(z)\right\}$ will be

$$
P_{n}(z)=\left(e^{i \delta} / \xi\right)^{n} f_{n}(z) \quad(n \geqq 0) .
$$

We shall prove that the transpose of this set is not effective in $D(C)$. In fact, if the transpose were effective in $D(C)$, then by Theorem 1 it would be effective in $\bar{D}(C)$. The following theorem asserts that this is not the case.

THEOREM 2. For all forms of the Faber curve $C$, and for all values of $\xi \geqq \beta$, the Cannon function of the transpose $\left\{\widetilde{P}_{n}(z)\right\}$ of the set $(6.3)$ is $\tilde{\kappa}(C)>\gamma$. Moreover, there are certain Faber curves for which $\tilde{\kappa}(C)=\sigma$. 
Proof. We first observe from (3.1) and (6.3) that

$$
\tilde{P}_{n}(z)=\tilde{f}_{n}\left(z e^{i \delta} / \xi\right)=\frac{\xi e^{-i \delta} \phi^{\prime}\left(\xi e^{-i \delta} / z\right)}{z\left\{\phi\left(\xi e^{-i \delta} / z\right)\right\}^{n+1}} \quad(n \geqq 0),
$$

and consequently, $z^{n}$ admits the representation

$$
z^{n}=\left(\xi e^{-i \delta}\right)^{n} \sum_{k=n}^{\infty} \bar{f}_{k, n} \widetilde{P}_{k}(z)
$$

Hence, (6.1) and (6.2) imply that

$$
\left|\widetilde{P}_{k}\left(z_{0}\right)\right|=\xi L /\left(\alpha \tau^{k+1}\right) \quad(k \geqq 0),
$$

where $L=\left|\phi^{\prime}\left(\xi e^{-i \delta} / z_{0}\right)\right|>0$.

Also, if we write

$$
m(r)=\inf \{|\psi(z)|:|z|=r\} \quad(r \geqq \alpha),
$$

and

$$
w_{0}=\xi e^{i(\omega-\delta)} / \eta, \quad \sigma_{1}=\left|\psi\left(w_{0}\right)\right|,
$$

it can be verified from (2.7), (5.2), (6.6) and (6.7) that

$$
\sigma_{1} \geqq m(\xi / \eta)>m(\alpha)=\gamma .
$$

We now consider the function $F(z)=1 /\left(w_{0}-z\right)$. In view of (5.2) and (6.7) it is easily seen that $F(z) \in H\left(C_{\sigma_{1}}\right)$ and that the Taylor expansion of $F(z)$ is valid in $\bar{D}(C)$. Suppose that the basic series associated with $F(z)$ is $\sum_{k} A_{k} \widetilde{P}_{k}(z)$, then according to Newns $[4,(3.5)$ and (3.9)], the basic coefficients $\left(A_{k}\right)$ can be calculated through (6.4). Hence, applying (6.7) we obtain

$$
A_{k}=\left(1 / w_{0}\right) \bar{f}_{k}\left(\eta e^{-i \omega}\right) .
$$

A combination of (1.5), (6.2), (6.5), (6.7) and (6.9) easily leads to the following inequality.

$$
\left|A_{k} \widetilde{P}_{k}\left(z_{0}\right)\right| \geqq(\eta L / \alpha \tau)\left\{1-(T /(\eta-T))(K / \tau)^{k}\right\}>(\eta L / 2 \alpha \tau) \quad \text { for } k>k_{0},
$$

where $K=\inf \{G(r): r>T\}$ (so that $K<\tau$ ), and $k_{0}$ is chosen so that

$$
(T /(\eta-T))(K / \tau)^{k}<1 / 2 \quad \text { for } k>k_{0} .
$$

It follows that the basic series associated with $F(z)$ diverges at the point $z_{0} \in C$. We conclude therefore that the set $\left\{\widetilde{P}_{n}(z)\right\}$ is not effective for $H\left(C_{\sigma_{1}}\right)$ in $\bar{D}(C)$, and so by $(6.8) \tilde{\kappa}(C) \geqq \sigma_{1}>\gamma$.

To complete the proof of the theorem, we observe that $\sigma_{1}=\sigma$ $=h(\xi / \eta)$ whenever we can arrange that $\left|\psi\left(w_{0}\right)\right|=\sigma$. That is to say, 
whenever the chosen numbers $\delta$ and $\omega$ can allow for $\left|\psi\left\{\xi e^{i(\omega-\delta)} / \eta\right\}\right|$ to be equal to $h(\xi / \eta)$. This is, in fact, the case (uniformly for $\xi \geqq \beta$ ) when $C$ is a circle, an ellipse or a Cassini oval. For such forms of the Faber curve $C$ the above procedure leads to the conclusion that $\tilde{\kappa}(C) \geqq \sigma$ and thus by Theorem $1, \tilde{\kappa}(C)=\sigma$. The proof of Theorem 2 is therefore complete.

7. Conclusion. In conclusion, we observe from (1.1) and (3.3) that

$$
\xi / \eta \leqq \alpha\{1-(2 J \alpha / \xi)\}^{-1},
$$

by taking $\xi$ large enough, where $J=\sup \{|\phi(t)-t|:|t| \geqq \gamma\}$. Hence (5.2) and (7.1) imply that

$$
\lim _{\xi \rightarrow \infty} \xi / \eta=\alpha .
$$

In collaboration with Theorem 1, the relation (7.2) asserts that the ultimate value, as $\xi$ tends to infinity, of the bound for the Cannon function $\tilde{\kappa}(C)$ of the transpose $\left\{\widetilde{P}_{n}(z)\right\}$ of the set $\left\{\xi^{-n} p_{n}(z)\right\}$ is

$$
h(\alpha)=h\{G(\gamma)\} .
$$

It should be noted, on the other hand, that the upper bound for the Cannon function $\lambda(C)$, of the square of the set $\left\{p_{n}(z)\right\}$ is (cf. Newns $[3$, Theorems 1, 3])

$$
G\{h(\gamma)\} .
$$

The values (7.3) and (7.4) coincide whenever $h(r)=G(r)$, as, for example in the case of the Cassini oval. It is instructive to find out that the equality

$$
\lambda(C)=\lim _{\xi \rightarrow \infty} \tilde{\kappa}(C)
$$

actually occurs for the set $\left\{p_{n}(z)\right\}$, given by $p_{n}(z)=i^{-n} f_{n}(z)$, where $\left\{f_{n}(z)\right\}$ is the $\phi$-base of the above curve. In fact, by direct calculation and by application of Theorem 2 above and Newns' already mentioned result, it can be easily shown that

$$
\lambda(C)=\left(c^{2}+2\right)^{1 / 2}
$$

and that

$$
\tilde{\kappa}(C)=\left\{\frac{\xi^{2}\left(c^{2}+2\right)-2\left(c^{2}+1\right)}{\xi^{2}-2\left(c^{2}+1\right)}\right\}^{1 / 2} \rightarrow\left(c^{2}+2\right)^{1 / 2} \quad \text { as } \xi \rightarrow \infty .
$$

Finally, I wish to express my warmest gratitude to the referee of this paper for his several useful and constructive suggestions. 


\section{REFERENCES}

1. G. Faber, Über die Polynomische Entwickelung, Math. Ann. 57 (1903), 389-408.

2. W. F. Newns, On the representation of analytic functions by infinite series, Philos. Trans. Roy. Soc. London. Ser. A 245 (1953), 429-468. MR 14, 968.

3. - The product of two simple sets effective in a Faber region, Nederl. Akad. Wetensch. Proc. Ser. A 63 = Indag. Math. 22 (1960), 187-191. MR 22 \#1685.

4. - The range of effectiveness of a basic set, Proc. London Math. Soc. (3) 18 (1968), 745-767. MR 38 \#328.

5. J.-M. Whittaker, Sur les séries de base de polynômes quelconques, GauthierVillars, Paris, 1939. MR 11, 344.

University of Assiut, Assiut, United Arab Republic 